\title{
Advances in the management of pediatric genitourinary rhabdomyosarcoma
}

\author{
Michael D. Deel \\ Department of Pediatrics, Division of Hematology/Oncology, Duke University School of Medicine, Durham, NC, USA \\ Correspondence to: Michael D. Deel, MD. Department of Pediatrics, Division of Hematology/Oncology, Duke University School of Medicine, Box \\ 102382 DUMC, Durham, NC, 27710, USA. Email: michael.deel@duke.edu.
}

\begin{abstract}
Recent clinical trials have revealed several unanticipated complexities in the optimal management of genitourinary rhabdomyosarcoma (RMS). Improvement in outcomes for low- and intermediate-risk RMS over the past several decades led to the design of clinical trials aimed at reducing acute and late toxicity from extirpative surgeries, conventional radiotherapy, and cytotoxic chemotherapy. Results from these studies are mixed and have illuminated areas where historical risk stratification strategies need refining. Although radiotherapy has now become the standard for local control for most patients with genitourinary RMS, recent studies are demonstrating that there may be opportunities to minimize radiation toxicity while maintaining acceptable failure-free survival. A reduction in cyclophosphamide exposure may benefit select low-risk RMS patients but recent results illustrate that decreasing therapy intensity for most genitourinary RMS patients will require careful consideration in future prospective trials. Finally, recent studies highlight differences in perspective between European and North American investigators regarding the optimal balance of increased local failure rates but less toxicity versus improved event-free survival at a cost of higher toxicity. This review focuses on the results from the most recent RMS clinical trials and discusses their implications for the management of pediatric genitourinary RMS.
\end{abstract}

Keywords: Genitourinary; rhabdomyosarcoma (RMS)

Submitted Jan 22, 2020. Accepted for publication May 21, 2020.

doi: 10.21037/tau-20-480

View this article at: http://dx.doi.org/10.21037/tau-20-480

\section{Introduction}

Rhabdomyosarcoma (RMS) comprises about $40 \%$ of all childhood soft tissue sarcomas, and while RMS makes up less than $1 \%$ of malignancies in adults, it accounts for approximately $8 \%$ of solid tumors in children (1). Histologically, RMS tumors are high-grade neoplasms originating from primitive mesenchymal tissue and resembling skeletal muscle histogenesis (2). RMS is comprised of four distinct histologic subtypes: (I) embryonal RMS, which are most common and typically occur in favorable sites in the head and neck and retroperitoneal regions of younger children $(3,4)$; (II) alveolar RMS, which represent less than a third of cases, frequently occur in unfavorable sites in the trunk or extremities of adolescents and young adults, and have characteristic recurrent $\mathrm{t}(2 ; 13)$ and $\mathrm{t}(1 ; 13)$ chromosomal translocations encoding PAX3/7FOXO1 fusion proteins (3-5); (III) spindle cell/sclerosing RMS, which is rare and may correlate with worse prognosis, but also has a distinct variant occurring in infants that is associated with $N C O A 2$ or $V G L L 2$ translocations and more favorable outcomes (2); and (IV) pleomorphic RMS, which is more aggressive and although extremely rare in children, comprises up to $43 \%$ of cases of RMS in adults (6). Overall, the incidence rate of RMS is approximately 4.5 per million individuals less than 20 years of age, accounting for about 350 new cases per year in the United States (1). The genitourinary tract represents the primary site for approximately $20-25 \%$ of RMS cases $(7,8)$. Genitourinary RMS can arise in favorable sites such as vagina or uterus (9), but often arise in the bladder or prostate, which are unfavorable sites (10). 
Table 1 Rhabdomyosarcoma TNM staging and clinical grouping

$\begin{array}{ll}\text { TNM staging } & \text { Non-invasive: tumor confined to organ or tissue of origin } \\ \text { T2 } & \text { Invasive: tumor extension beyond the organ or tissue of origin } \\ \mathrm{N}_{0} & \text { No regional lymph node involvement } \\ \mathrm{N}_{1} & \text { Regional lymph node involvement } \\ \mathrm{N}_{\mathrm{X}} & \text { Regional lymph nodes not examined } \\ \mathrm{M}_{0} & \text { No distant metastasis } \\ \mathrm{M}_{1} & \text { Distant metastatic disease } \\ \text { Clinical grouping } & \text { Localized tumor, completely removed with microscopically clear margins and no regional lymph node involvement. } \\ \text { I } & \text { Localized tumor, completely removed with: (a) microscopic residual disease; (b) regional disease with involved, grossly } \\ \text { II } & \text { removed regional lymph nodes; or (c) regional disease with involved nodes, grossly removed but with microscopic residual } \\ & \text { and/or histologic involvement of the most distal node from the primary tumor } \\ \text { III } & \text { Localized tumor, incompletely removed with gross, residual disease after: (a) biopsy only or (b) subtotal resection } \\ \text { IV } & \text { Orbit; nonparameningeal head and neck; genitourinary tract other than kidney, bladder, and prostate; biliary tract }\end{array}$

Favorable sites: orbit; nonparameningeal head and neck; genitourinary tract other than kidney, bladder, and prostate; biliary tract.

Due to the implementation of risk stratification and standardized multimodal management through cooperative clinical trials, outcomes for patients with RMS have improved substantially over the past $30+$ years and survival for patients with RMS now exceeds $70 \%$ overall and $80 \%$ for patients with genitourinary RMS (11). Improved overall survival rates have led to a renewed emphasis on reducing local control morbidity and chemotherapy toxicity. However, historical differences exist between cooperative groups with regard to approach in local control measures for genitourinary RMS (9,12-15). Moreover, recent clinical trials aimed at minimizing toxicity for low- and intermediate-risk RMS by decreasing chemotherapy and/or radiotherapy exposure demonstrated inferior local control rates $(16,17)$. On the other hand, improved overall survival was demonstrated with the addition of maintenance therapy following standard induction therapy in patients with nonmetastatic intermediate-risk RMS (18). For patients with high-risk metastatic RMS, intensifying chemotherapy using an interval compressed multi-agent approach failed to improve overall survival (19). Indeed, with a growing appreciation for how the complexities of patient characteristics, tumor size, anatomic location, disease stage, and histologic and molecular features impact the clinical behavior of genitourinary RMS, optimal treatment strategies are still evolving.

\section{Advances in RMS risk stratification}

Historically, risk stratification for RMS, as employed by each of the major prior and current cooperative study groups [Intergroup Rhabdomyosarcoma Study Group (IRSG), Children's Oncology Group (COG), International Society of Pediatric Oncology (SIOP) Malignant Mesenchymal Tumor Group (MMT), European pediatric Soft tissue sarcoma Study Group (EpSSG), Cooperative Weichteilsarkom Studiengruppe der GPOH Group (CWS), and Italian Cooperative Soft Tissue Sarcoma Group (ICG)] takes into account presurgical (TNM) staging as well as postsurgical clinical grouping. TNM staging is based on the anatomic location and invasiveness of the primary tumor, tumor size, nodal status, and extent of metastasis (20). The clinical grouping is based on the extent of residual tumor after the initial resection as well as local nodal involvement and distant spread (21) (Tables 1,2). Each classification system is individually prognostic $(4,8)$ and combine to allow patients to be stratified into low-, intermediate-, or highrisk groups (COG) $(3,22)$ (Table 3), or low-, standard-, high-, very high-, and metastatic-groups (EpSSG) (23) (Table 4). The major difference between the COG and EpSSG classifications is that most patients considered intermediate-risk per COG are classified as high-risk per EpSSG, as any lymph node positivity is considered high- 
Table 2 Rhabdomyosarcoma pretreatment staging system

\begin{tabular}{lccccc}
\hline Stage & Primary site & TNM stage & Tumor size & Regional nodes & Distant metastasis \\
\hline 1 & Favorable & T1 or T2 & Any size & $\mathrm{N}_{0}$ or $\mathrm{N}_{1}$ or $\mathrm{N}_{\mathrm{x}}$ & $\mathrm{N}_{0}$ \\
2 & Unfavorable & T1 or T2 & $\leq 5 \mathrm{~cm}$ & $\mathrm{~N}_{0}$ or $\mathrm{N}_{\mathrm{x}}$ & $\mathrm{N}_{1}$ \\
3 & Unfavorable & T1 or T2 & $\leq 5 \mathrm{~cm}$ & $\mathrm{~N}_{0}$ or $\mathrm{N}_{1}$ or $\mathrm{N}_{\mathrm{x}}$ & $\mathrm{M}_{0}$ \\
& & & $>5 \mathrm{~cm}$ & $\mathrm{~N}_{0}$ or $\mathrm{N}_{1}$ or $\mathrm{N}_{\mathrm{x}}$ & $\mathrm{M}_{1}$ \\
\hline
\end{tabular}

Table 3 Rhabdomyosarcoma risk stratification per COG*

\begin{tabular}{lccc}
\hline Risk group & FOXO1 fusion status & Stage & Group \\
\hline Low & Negative & 1 & I, II, III (orbital only) \\
Intermediate & Negative & 2 & I, II \\
& & 1 & III (nonorbital) \\
& & 2,3 & III I, II \\
& & 3 & IV (age <10 years) \\
High & Positive & 4 & I, II, III \\
& Negative & $1,2,3$ & IV (age $>10$ years) \\
\hline
\end{tabular}

*, based on ongoing intermediate-risk phase III COG trial ARST1431.

Table 4 Rhabdomyosarcoma risk stratification per EpSSG*

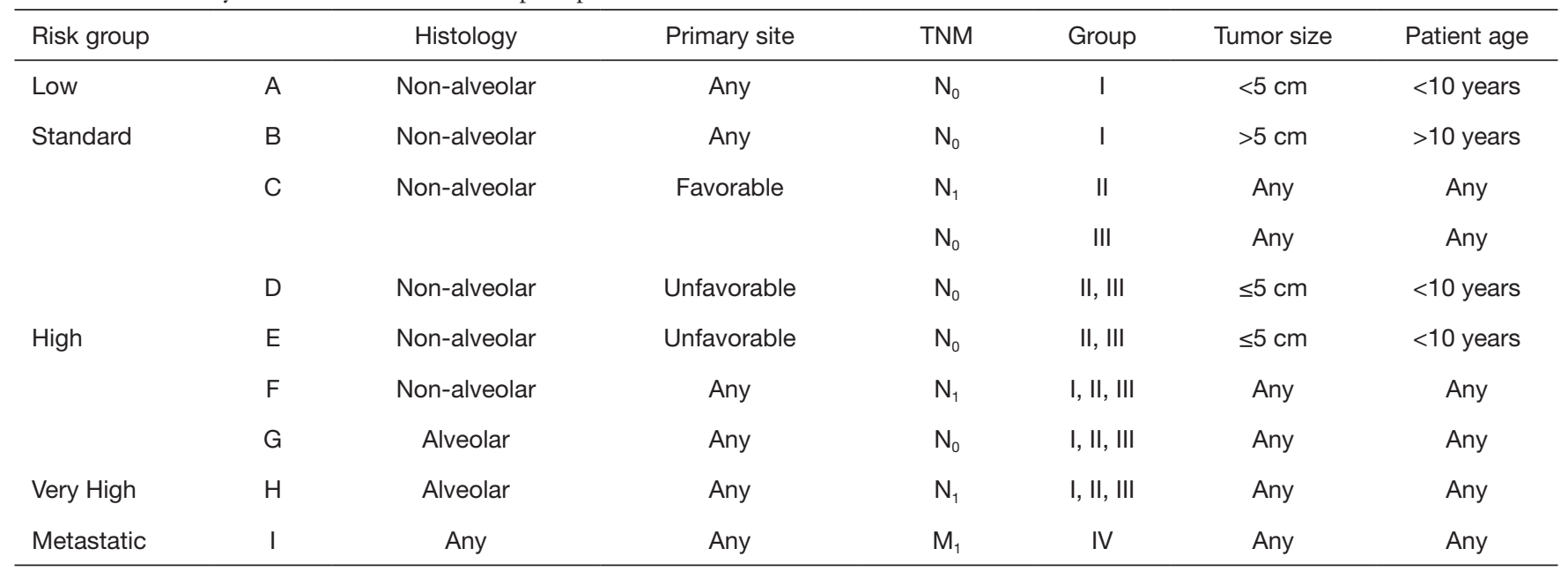

*, based on EpSSG trial RMS-2005. 
Table 5 Overall survival rates based on molecular and clinical features

\begin{tabular}{|c|c|c|c|}
\hline Molecular and clinical feature & Overall survival rate & Localized & Metastatic \\
\hline Low MG5 score & $\sim 90 \%$ & & \\
\hline High MG5 score & $\sim 50 \%$ & & \\
\hline \multicolumn{4}{|c|}{ "Oberlin-defined" clinical indicators for metastatic rhabdomyosarcoma } \\
\hline$>2$ Oberlin risk factors & $14 \%$ & & \\
\hline \multicolumn{4}{|l|}{ FOXO1 fusion status* } \\
\hline Fusion-negative & & $88 \%$ & $58 \%$ \\
\hline Fusion-positive & & $65 \%$ & $19 \%$ \\
\hline \multicolumn{4}{|c|}{$\begin{array}{l}\text { *, 5-year overall survival based on FOXO1 fusion status and extent of tumor spread; ", estimated 5-year overall survival retrospectively } \\
\text { evaluated using } 5 \text { gene signature: EPHA2 (Ephrin Receptor A2), EED (Embryonic Ectoderm Development), NSMF (NMDA receptor } \\
\text { synaptonuclear signaling and neuronal migration factor), CBS (Cystathionine- } \beta \text {-synthase), and EPB41L4B (Erythrocyte Membrane Protein } \\
\text { Band } 4.1 \text { like 4B); }{ }^{\$}, 3 \text {-year overall survival for metastatic rhabdomyosarcoma based on "Oberlin-defined" negative clinical risk factors: } \\
\text { patient age }<1 \text { year or }>10 \text { years, } \geq 3 \text { metastatic sites, bone marrow involvement. }\end{array}$} \\
\hline
\end{tabular}

risk by EpSSG (23). The prognostic significance of RMS risk stratification is well established. Outcomes for low-risk localized RMS are outstanding, with over $90 \%$ of patients achieving cure $(3,8)$. On the other hand, 5 -year overall survival rates for high-risk metastatic RMS remain below $30 \%$ and have not significantly improved over the past several decades (4).

More recently, the COG and European cooperative groups have expanded risk stratification to also include other prognostic indicators. From a multivariate analysis on 788 cases of metastatic RMS from nine separate studies by COG and European cooperative groups between 1984 and 2000, Oberlin and colleagues proposed four additional independent prognostic indicators ("Oberlin risk factors") that correlate with worse outcomes: age below 1 year or above 10 years, unfavorable primary tumor location, having three or more metastatic sites, and bone marrow involvement (4). Patients with metastatic RMS who had fewer than two Oberlin risk factors had an event-free survival (EFS) of $44 \%$ at 3 years, whereas those with two or more risk factors had an EFS of only $14 \%$ at 3 years (Table 5 ).

While these clinical indicators aid in delineating risks for metastatic disease, molecular features may advance the ability to distinguish risks groups for low- and intermediaterisks RMS. Several retrospective studies indicate that the presence of characteristic fusion oncoproteins comprising the majority of histologic alveolar RMS cases is prognostic. Although fusion-negative alveolar RMS has an indistinguishable molecular profile and similar clinical behavior to embryonal RMS (24), alveolar cases with $P A X 3$ FOXO1 or PAX7-FOXO1 fusions are associated with worse outcomes $(5,25,26)$. A recent study examining over 1,700 low-, intermediate-, and high-risk patients from six previous COG clinical trials from 1997 to 2013 determined that after metastatic disease, FOXO1 fusion status is the most important prognostic factor. In this study, the 5 -year overall survival of patients with fusion-negative $v s$. FOXO1-fusion positive RMS was $88 \%$ vs. $65 \%$ for localized disease and $58 \%$ vs. $19 \%$ for metastatic disease (27) (Table 5). Both the COG in ARST1431 and EpSSG are prospectively using fusion gene status instead of histology in new clinical trials $(28,29)$. The prognostic utility of more robust molecular classifications beyond just fusion status is also being assessed (30,31). A retrospective evaluation of intermediate-risk fusion-negative RMS using a five gene metagene signature, termed MG5, strongly correlates with outcomes $(5,31)$ (Table 5). Evaluation of MG5 in large prospective trials may identify subgroups that warrant modifications to therapy intensity.

New risk stratification systems that more stringently define low- and high-risk groups have been proposed $(27,32)$. Recent COG clinical trials attempting to deescalate therapy for low- or intermediate-risk RMS $(16,17)$ or to intensify therapy for high-risk metastatic RMS (19) have brought to light that responses to treatment based on risk stratification is more nuanced than previously believed. 
Results from these trials have specific implications for genitourinary RMS.

\section{Genitourinary RMS}

Genitourinary RMS accounts for $20-25 \%$ of all RMS cases and includes involvement of the bladder, prostate, paratesticular areas, vagina, uterus, and occasionally the kidney or ureter $(8,33)$. Approximately $90 \%$ of cases are embryonal histology, and 5-year overall survival for all genitourinary RMS exceeds $80 \%$ (11). There is a biomodal age distribution with the majority of new cases occurring in toddlers aged 2-4 years or adolescents aged 15-19 years (7). Younger children more commonly get a variant of embryonal RMS, either sarcoma botyroides, which is a polyploid tumor that occurs in the bladder or vagina and appears as a "cluster of grapes" (34), or spindle cell RMS, which often occurs in the paratesticular region. Both botryoid and spindle cell subtypes have excellent outcomes, with 5 -year overall survival rates approaching $90 \%$ (10).

Most cases of genitourinary RMS occur sporadically, although RMS is associated with several genetic syndromes. Patients with Li-Fraumeni syndrome, which is caused by loss-of-function germline mutations in TP53, are predisposed to malignancy, including an $18-27 \%$ prevalence of RMS $(35,36)$. Costello syndrome, a rare overgrowth syndrome caused by germline mutations in HRAS, is associated with an estimated $10 \%$ lifetime risk of embryonal RMS (37). Genitourinary RMS is also reported to occur with increased frequency for patients with DICER1 syndrome (38), and RMS is associated with several other cancer predisposition syndromes such as Beckwith-Wiedemann, neurofibromatosis type 1, Gorlin's basal cell nevus, and Rubinstein-Taybi syndromes (39). Consideration for genetic counseling referral should be given to genitourinary RMS patients, particularly younger children, or those who present with syndromic features or a family history of malignancy (40).

Regarding risk stratification, non-bladder/non-prostate sites are considered favorable and can therefore only be stage 1 or stage 4 , depending on the absence or presence of distant metastasis. In contrast, bladder and prostate RMS represent unfavorable sites and are thus never stage 1 $(4,8,21)$. Using the historical IGSG/COG risk stratification schema, most paratesticular and female genital tract RMS have been treated as low-risk, whereas bladder or prostate RMS cases have typically been treated as intermediaterisk $(4,8,21)$. The excellent overall survival rates for genitourinary RMS have led to an emphasis on reducing morbidity through organ preservation and decreased chemotherapy and radiotherapy toxicity. However, precisely identifying which patients may benefit from less aggressive local control measures or a reduction in therapy without compromising EFS or long-term survival based has been challenging.

\section{Advanced approaches to local control for genitourinary RMS}

When it is possible to achieve negative microscopic margins with minimal morbidity, resection of the primary tumor is the preferred method of local control (3). However, this is often not possible for genitourinary RMS. Although the presence of residual tumor prior to starting adjuvant therapy shifts patients from IRS group I or II to IRS group III, aggressive exenterative up front surgeries are no longer recommended due to significant long-term morbidities such as urinary diversion, infertility, and sexual dysfunction (41). In the 1970 s, the mainstay local control approach for bladder and prostate RMS included anterior exenteration followed by chemotherapy and possibly radiotherapy. Using this approach, the first IRSG study demonstrated $78 \% 5$-year overall survival, but $77 \%$ of patients had loss of bladder function (21). Subsequent ISRG studies using neoadjuvant chemotherapy and radiotherapy have improved bladder preservation rates to above $60 \%$ without compromising EFS or overall survival $(8,42)$ (Table 6). Therefore, unless organ/bladder preservation with complete resection of the RMS tumor can be achieved, the standard of care today is typically multimodal strategy of a minimally invasive initial biopsy, neoadjuvant chemotherapy and radiotherapy, with or without a delayed surgical excision $(10,43)$. One notable exception to the initial surgical management of genitourinary RMS exists. Patients 10-year of age or older with paratesticular RMS have a substantial risk of nodal relapse, which is only $8 \%$ in boys less than 10 -year of age but $31 \%$ in older males (44) (Table 6). Therefore, ipsilateral retroperitoneal lymph node evaluation is recommended for males older than 10-year of age and children younger than 10-year who have large paratesticular RMS greater than $5 \mathrm{~cm}$. The extent of resection, whether total sampling is adequate or total retroperitoneal lymph node dissection is needed, is still not definitively established (44-46).

A standard for the implementation, timing, dosing, or modality of adjuvant radiotherapy for local control 
Table 6 Special local control considerations for genitourinary rhabdomyosarcoma

\begin{tabular}{|c|c|}
\hline Local control consideration & Rates \\
\hline Upfront exenteration followed by chemotherapy +/- radiotherapy & $23 \%$ \\
\hline Minimally invasive biopsy with neoadjuvant chemotherapy and radiotherapy & $>60 \%$ \\
\hline \multicolumn{2}{|l|}{ Rates of nodal relapse in paratesticular rhabdomyosarcoma } \\
\hline Males $\geq 10$ years of age & $31 \%$ \\
\hline \multicolumn{2}{|l|}{ 10-year event-free survival rates for vaginal and uterine rhabdomyosarcoma } \\
\hline With radiotherapy & $71 \%$ \\
\hline Without radiotherapy & $81 \%$ \\
\hline Protocol-specified radiotherapy & $77 \%$ \\
\hline No local therapy & $46 \%$ \\
\hline Delayed local therapy & $62 \%$ \\
\hline Local therapy not delayed & $78 \%$ \\
\hline
\end{tabular}

of genitourinary RMS is also still evolving. Most of the European trials have attempted to delay or omit systemic radiotherapy and have been willing to tolerate lower EFS and higher rates of relapse in order to minimize late radiotherapy-related toxicities (14). For patients with vaginal or uterine RMS who achieved a complete remission with chemotherapy alone, the SIOP group in the MTT 89 study opted for no local treatment (47-49). On the other hand, the COG adopted a more aggressive systemic radiotherapy approach in response to a 2011 report from ARST0331 of high local failure rates in non-completely resected vaginal RMS with elimination of radiotherapy $(9,50,51)$. An important caveat to interpreting results from ARST0331 is that total cumulative akylator dosing had also been lowered, which may have contributed to the observed $43 \%$ local recurrence rate compared to only $26 \%$ on the COG predecessor trial D9602.

A recent study retrospectively examined outcomes for 237 patients with vaginal and uterine RMS treated on several cooperative group (COG, SIOP, ICG and EpSSG) trials from 1981 to 2009 . In this study, $23 \%$ to $46 \%$ of patients received radiotherapy, but European groups used intracavitary brachytherapy $64 \%$ to $88 \%$ of the time compared to only $23 \%$ in the COG trials. The 10 -year
EFS and overall survival for all patients were $74 \%$ and $92 \%$, respectively. Elimination of initial radiotherapy did not impact 10 -year overall survival, which was $89 \%$ with radiotherapy and $94 \%$ without radiotherapy. However, confirming the observations from ARST0331, patients who did not receive radiotherapy had a lower EFS of 71\%, compared to $81 \%$ EFS for those receiving radiotherapy (14) (Table 6).

Standard dosing of conventional radiation takes into account several clinical factors, including primary site, tumor size, involvement of lymph nodes, extent of resection, and FOXO1 fusion status. A dose of 36 Gy to the primary site may be sufficient for completely resected fusion positive RMS tumors, whereas incompletely resected tumors require 50.4 Gy (50). Bulky intermediate-risk RMS greater than $5 \mathrm{~cm}$ in size remains a challenge with local recurrence rates of $25 \%$, which has not improved over the past several COG trials (50). Higher doses of radiotherapy to 59.4 Gy for these cases is being prospectively evaluated in ARST1431. Obtaining adequate local control for infants with genitourinary RMS also continues to present a challenge. Infants with RMS below 12 months of age have historically had worse outcomes compared to older toddleraged children $(52,53)$, which may be due to physicians 
delaying or lessening therapy in order to reduce toxicity in developing neonates (54-56). A study from the ICG reported high local failure rates of $54 \%$ when radiotherapy was omitted for young age (55). A recent report from the COG that evaluated outcomes for infants on ARST0331 and ARST0531 demonstrated that $43 \%$ of infants received individualized non-protocol directed radiotherapy in which radiation was typically delayed or omitted. Those receiving individualized therapy had higher rates of local failure and 5 -year EFS, which was $55.6 \%$ with individualized local therapy versus $77.5 \%$ with protocol-specified therapy (54) (Table 6).

Results from these recent clinical trials demonstrate the challenge and differences in philosophy of balancing the risks of long-term toxicity while also not compromising local control and EFS or overall survival. These studies also confirmed that better local control measures are needed for bulky tumors greater than $5 \mathrm{~cm}$ and for infants with RMS. Although conventional external beam radiation remains the standard, alternative radiotherapy modalities are being evaluated. Compared to conventional fractioning of radiation, hyperfractionating radiotherapy has been shown to offer no benefit to EFS or overall survival in children with IRS group III RMS (57). Experience using intracavitary brachytherapy for primary local control, albeit with small sample sizes, suggests this may be an effective modality. Of the 28 female genital tract RMS patients who received brachytherapy alone for local control, only two experienced local recurrence (14). Similarly, using brachytherapy as primary control for a cohort of 26 males with prostate or bladder cancer allowed patients to maintain continence with only one local and one metastatic relapse at a medium follow of 4-year (58). Proton therapy is also a promising alternative $(59,60)$. A recent study of 83 RMS patients who received proton therapy, which only included four genitourinary non-bladder/prostate RMS cases, reported $78 \%$ EFS and improved quality of life measures compared to historical controls (61). In a small study of seven children with prostate or bladder RMS, treatment with proton therapy allowed for decreased radiation exposure to surrounding normal structures, although longterm outcome data is limited (62). Finally, for patients with intermediate-risk RMS, delayed primary excision may facilitate a decrease in radiation while still maintaining local control rates similar to historical controls (63).

For infants and for individuals with unresected vaginal RMS, future clinical trial design and treating physicians must balance the risks of late effects from radiotherapy exposure against the risks for disease recurrence necessitating additional morbidity from cytotoxic chemotherapy, radiotherapy and surgery. Future prospective clinical trials should also elucidate whether brachytherapy, proton therapy, and/or delayed primary excision are able to minimize late effects while maintaining high local control rates.

\section{Advances in chemotherapy for genitourinary RMS}

While eradication of the gross primary tumor through a combination of surgery and/or radiotherapy is essential for most RMS cases, achieving cures also requires systemic chemotherapy. Early studies from the 1960s and 1970s demonstrated high rates of regional and metastatic recurrence for RMS patients not receiving chemotherapy (64). More recently, sensitive molecular testing with RTPCR confirmed a suspicion that even localized tumors present with micrometastatic disease. RMS cells were detected in sampling of the peripheral blood or bone marrow of 12 of 16 cases of grossly localized RMS (65). Therefore, unlike the management of non-RMS soft tissue sarcomas, standardized care for all RMS cases, even small tumors achieving gross total resection, includes adjuvant chemotherapy.

The standard first-line chemotherapy for RMS is an intensive alkylator-containing regimen in combination with vincristine and actinomycin $\mathrm{D}$. The alkylator used in North America is cyclophosphamide (combination VAC), whereas ifosfamide (combination IVA) is typically given in Europe. A randomized study comparing VAC versus IVA for initial therapy followed by VAC for all patients demonstrated these two regimens are equivalent (8). For intermediaterisk RMS, which encompasses about $60 \%$ of all newly diagnosed cases, cycles of VAC alternating with vincristine and irinotecan (VI) is also accepted as a standard backbone (VAC/VI) (32). The COG study ARST0531, which randomized intermediate-risk RMS patients to receive VAC or VAC/VI demonstrated comparable $65 \%$ to $68 \%$ 4-year EFS between the two arms (66). However, VAC/ VI was associated with less hematologic toxicity and fewer hospitalizations; and with a $50 \%$ reduction in cumulative alkylator exposure, VAC/VI may also decrease the risks for hemorrhagic cystitis, infertility and secondary malignancy.

\section{Low-risk genitourinary RMS}

In attempt to preserve gonadal function, the most recent 
Table 7 Summary of outcomes for low-risk rhabdomyosarcoma

\begin{tabular}{ll}
\hline Comparison of event-free survival from D8602 vs. ARST0331* & FFS \\
\hline D8602 (28 $\mathrm{g} / \mathrm{m}^{2}$ cumulative cyclophosphamide) & $85 \%$ \\
ARST0331 (4.8 g/m ${ }^{2}$ cyclophosphamide) & $64 \%$ \\
3-year survival rates per ARST0331 subsets I and II & $89 \%$ \\
Subset I & $70 \%$ \\
Subset II & $57 \%$ \\
Females with genitourinary tract RMS ${ }^{*}$ & $98 \%$ \\
\hline
\end{tabular}

FFS, failure-free survival; OS, overall survival. *, represented EFS is 5-year for D8602 and 3-year for ARST0331; ", females with genitourinary tract rhabdomyosarcoma also did receive local radiotherapy.

low- and intermediate-risk COG trials, ARST0331 and ARST0531, respectively, attempted to lower cumulative cyclophosphamide exposure without compromising outcomes. Using standard VAC therapy, low-risk patients have at least an $83 \% 5$-year EFS and 95\% overall survival $(8,47)$. Historical IRSG risk stratification considered lowrisk RMS to be localized favorable histology RMS tumors that either arise in favorable primary sites or arise in unfavorable primary sites but are grossly resected (either stage 1 embryonal RMS or stage $2 / 3$ group I/II embryonal RMS) (3). Based on results of the predecessor low-risk trial D9602, two subsets were evaluated in ARST0331: subset I (stage 1/2 group I/II or stage 1 group III orbital) or subset II (stage 1 group III nonorbital or stage 3 group I/II). Subset I was treated with only 24 weeks of chemotherapy that included four cycles of VAC followed by four cycles of VA. The total cumulative cyclophosphamide dose was reduced to $4.8 \mathrm{~g} / \mathrm{m}^{2}$. For this subset, outstanding outcomes were maintained using less intensive therapy, with 3-year failurefree and overall survival at $89 \%$ and $98 \%$, respectively (67) (Table 7).

Low-risk subset II patients had seen an improvement with IRS-IV over IRS-III with the addition of cumulative cyclophosphamide dosing of $26.4 \mathrm{~g} / \mathrm{m}^{2}$ (8). In ARST0331, subset II received a reduction in cumulative cyclophosphamide dosing to $4.8 \mathrm{~g} / \mathrm{m}^{2}$ with four VAC cycles followed by 12 cycles of VA over 46 weeks. Importantly, females with genitourinary tract RMS who had a complete response to chemotherapy with or without delayed resection did not receive radiotherapy. Although excellent 3 -year overall survival rates of $92 \%$ were achieved, 3-year failure-free survival was much lower than anticipated: $70 \%$ overall and only $57 \%$ for the 21 patients with genitourinary tract tumors (16). Compared to D8602, which used $28 \mathrm{~g} / \mathrm{m}^{2}$ cyclophosphamide and reported 5-year EFS of $85 \%$, EFS was only $64 \%$ with lower cyclophosphamide dosing (16) (Table 7). Although the study investigators concluded that these recurrence rates are suboptimal, it again highlights the aforementioned difference in prospective between North American and European cooperative groups, in which some European groups are often more willing to accept local recurrence rates in order to decrease morbidity for the majority of patients who receive treatment if overall survival rates are not jeopardized (47).

\section{Intermediate-risk genitourinary RMS}

In the phase III ARST0531 study, 424 patients with intermediate-risk RMS (stage 1-3, group I-III, nonmetastatic alveolar RMS at any primary site, or stage 2-3, group III incompletely resected embryonal RMS in an unfavorable site), including 55 patients with bladder/ prostate RMS, were randomized to receive either VAC or VAC/VI with cumulative cyclophosphamide doses of 16.8 or $8.4 \mathrm{~g} / \mathrm{m}^{2}$, respectively (17). This study was designed to enhance local control by delivering radiotherapy earlier at week 4 , and in the experimental arm, by giving radiotherapy concurrently with irinotecan which is reported to be a radiosensitizer (68). Radiotherapy guidelines on ARST0531 did not differ from D9803 except for allowing a cone down for tumors displaying a rapid response with 36 Gy. In an effort to increase enrollment, children below 24 months of age were encouraged to receive on-protocol radiotherapy but were allowed to receive individualized local control plans. Similar to what was observed with subset II low-risk RMS, a reduction in cumulative cyclophosphamide dosing was associated with inferior outcomes compared to D9803 $(17,50,66)$. 
Table 8 Summary of 5-year cumulative incidence of local failure in intermediate-risk rhabdomyosarcoma

\begin{tabular}{lll}
\hline Group & D9803 & ARST0531 \\
\hline Group I/II & $8.6 \%$ & $13.4 \%$ \\
Group III embryonal RMS & $19.4 \%$ & $27.9 \%$ \\
Group III alveolar RMS & $17.7 \%$ & $20.2 \%$ \\
Non-parameningeal sites & $15.6 \%$ & $25.2 \%$ \\
\hline
\end{tabular}

ARST0531 was associated with higher failure free rates, which was most pronounced in the group III embryonal RMS patients whose 5-year cumulative incidence of local failure was $27.9 \%$ compared to $19.4 \%$ on D9803. Multivariable analysis also demonstrated worse 5-year EFS and overall survival on ARST0531 compared to D9803 (17) (Table 8). Interestingly, local failure rates were not different between the VAC $\left(16.8 \mathrm{~g} / \mathrm{m}^{2}\right.$ cyclophosphamide) and VAC/ VI $\left(8.4 \mathrm{~g} / \mathrm{m}^{2}\right)$ cyclophosphamide) treatment arms, nor with the two treatment arms of D9803 $\left(25.1\right.$ vs. $30.8 \mathrm{~g} / \mathrm{m}^{2}$ cyclophosphamide) (50), which demonstrates that the optimal cyclophosphamide dose to achieve local control while also minimizing toxicity in intermediate-RMS is not yet established. The method of local control for traditionally resectable tumors such as bladder dome, extremity or trunk, was a notable difference between D9803, where $45 \%$ of patients received primary resection after induction chemotherapy, and ARST0531 in which only $17 \%$ of tumors were resected. However, investigators accounted for these differences in their regression models and outcomes were still worse on ARST0531 (17).

\section{Maintenance therapy for intermediate-risk genitourinary RMS}

The EpSSG recently reported on a phase III study, RMS 2005, evaluating the efficacy of adding 24 weeks of maintenance therapy with vinorelbine and low-dose continuous oral cyclophosphamide to non-metastatic highrisk (mostly intermediate-risk per IRS/COG) RMS patients who had achieved a complete response following 27 -week of therapy. In total, 371 enrolled patients between 2006 and 2016 were randomly assigned to either stop therapy or to receive an additional 24-week of maintenance therapy. The additional chemotherapy, which resulted in a modest increase in cyclophosphamide exposure $\left(4.2 \mathrm{~g} / \mathrm{m}^{2}\right)$ was well tolerated and was associated with improved disease-free and
Table 9 Summary of outcomes with maintenance therapy for intermediate-risk rhabdomyosarcoma

\begin{tabular}{lll}
\hline Group & EFS & OS \\
\hline Control group & $72.3 \%$ & $77.4 \%$ \\
Received maintenance therapy & $78.4 \%$ & $87.3 \%$ \\
\hline
\end{tabular}

EFS, event-free survival; OS, overall survival 3-year following randomization.

overall survival, which were $78.4 \%$ and $87.3 \%$, respectively, in the treatment arm compared to $72.3 \%$ and $77.4 \%$ in the control group at 3-year after randomization (18) (Table 9). These excellent results, which were only recently published, may lead to a universal change in standard management to include maintenance chemotherapy for all patients with intermediate- and possibly high-risk RMS.

\section{Ongoing clinical trials for low- and intermediate- risk RMS}

Lessons learned from the last COG low- and intermediaterisks studies as well as EpSSG RMS 2005 were incorporated into the ongoing phase III intermediate-risk COG study ARST1431. Previous cases of subset II low-risk RMS are reclassified as intermediate-risk, in which all patients receive $8.4 \mathrm{~g} / \mathrm{m}^{2}$ cyclophosphamide in a VAC/VI backbone. A study question as to whether the addition of temsirolimus, which has shown activity in relapsed RMS (28), will improve outcomes when included up front will be evaluated by randomization to VAC/VI or VAC/VI plus temsirolimus. Following completion, participants from both arms will continue to receive an additional 6 months of maintenance therapy. Also, because patients less than 10-year of age with group IV embryonal RMS have a reasonably good 64\% 3-year EFS (69), these individuals and are now being treated as intermediate-risk rather than high-risk. Finally, patients with bulky tumors larger the $5 \mathrm{~cm}$ will receive higher radiotherapy doses to 59.4 Gy instead of 50.4 Gy.

\section{High-risk genitourinary RMS}

Management for high-risk metastatic RMS continues to be a challenge with minimal improvement in outcomes over the past several decades. EpSSG RMS 2005 also investigated adding doxorubicin to their standard IVA backbone for high-risk RMS but this failed to lead to an 
improvement in EFS (70). The COG recently completed a phase II study, ARST0431, incorporating an interval compression strategy of alternating cycles of vincristine, doxorubicin, and cyclophosphamide with ifosfamide and etoposide (VDC/IE) into a VAC/VI backbone (19). Compared to historical controls, outcomes were improved with a 3-year EFS of $69 \%$ for only a subset of patients with one or no Oberlin-defined risk factors (4), but the majority of patients had more than one Oberlin risk factor and no benefit to increasing the intensity of therapy was demonstrated for this group (19). In the absence of available clinical trials, no universal consensus for the management of high-risk RMS exists (32), although select patients with limited Oberlin risks factors may benefit from escalated therapy.

\section{Relapsed genitourinary RMS}

Patients with recurrent RMS have a dismal prognosis with no direct comparisons of salvage regimens and no widely accepted standard of care to guide management. Unfortunately, about one third of RMS will relapse and the 5-year progression-free survival for all relapsed RMS patients is a dismal $17 \%(71,72)$. Approximately $95 \%$ of relapses will occur within 3-year of diagnosis and RMS has a median time to progression of 13 months from diagnosis. Metastatic recurrence occurs in about $40 \%$ of relapses and, compared to local or regional recurrence, is associated with a worse prognosis (73). Potential salvage regimens were recently reviewed elsewhere by a panel of RMS experts who recommend, in the absence of an available clinical trial, consideration of first-line salvage therapy for patients who are heavily pretreated with a combination of cyclophosphamide, vinorelbine, and temsirolimus, or giving VDC/IE and/or an irinotecan-based therapy for patients who did not initially receive intensive therapy (32). Breakthroughs for high-risk metastatic and recurrent RMS are desperately needed. Despite promising preclinical data, recent early phase trials of novel agents such as anti-IGF$1 \mathrm{R}$ and multi-tyrosine kinase inhibitor sorafenib have not shown single-agent activity in relapsed patients (74-76). Lack of improvement in the management for these patients is understandably a major emphasis for ongoing basic and translational studies.

\section{Conclusions}

Management for pediatric patients with genitourinary
RMS is complex and requires a multidisciplinary approach. Multimodal therapy in the context of cooperative clinical trials has led to substantial improvement in cure rates for pediatric genitourinary RMS, which now exceeds $80 \%$ (11). The excellent overall survival rate for low-risk RMS patients presents an opportunity to potentially decrease toxicity, but this may come at a cost of increased rates of local failure and recurrence. Additional prospective studies are required to delineate the optimal dosing and modalities for surgery, chemotherapy, and radiation for low- and intermediate-risk genitourinary RMS. Experience with alternative irradiation modalities, such as external beam proton therapy or nonradical surgery plus brachytherapy, is limited but may lessen long-term toxicities of bladder and sexual dysfunction. While the addition of maintenance therapy improves EFS and overall survival for intermediate-risk RMS, outcomes for high-risk metastatic RMS remain very poor.

\section{Acknowledgments}

Funding: This work was supported by a V Foundation Scholar award and Doris Duke Charitable Foundation award.

\section{Footnote}

Provenance and Peer Review: This article was commissioned by the Guest Editors (John Wiener, Jonathan Routh and Nicholas Cost) for the series "Pediatric Urologic Malignancies" published in Translational Andrology and Urology. The article was sent for external peer review organized by the Guest Editors and the editorial office.

Peer Review File: Available at http://dx.doi.org/10.21037/ tau-20-480

Conflicts of Interest: The author has completed the ICMJE uniform disclosure form (available at http://dx.doi. org/10.21037/tau-20-480). The series "Pediatric Urologic Malignancies" was commissioned by the editorial office without any funding or sponsorship. MDD reports grants from V Foundation and Doris Duke Charitable Foundation, during the conduct of the study. The author has no other conflicts of interest to declare.

Ethical Statement: The author is accountable for all aspects of the work in ensuring that questions related to the accuracy or integrity of any part of the work are 
appropriately investigated and resolved.

Open Access Statement: This is an Open Access article distributed in accordance with the Creative Commons Attribution-NonCommercial-NoDerivs 4.0 International License (CC BY-NC-ND 4.0), which permits the noncommercial replication and distribution of the article with the strict proviso that no changes or edits are made and the original work is properly cited (including links to both the formal publication through the relevant DOI and the license). See: https://creativecommons.org/licenses/by-nc-nd/4.0/.

\section{References}

1. Ognjanovic S, Linabery AM, Charbonneau B, et al. Trends in childhood rhabdomyosarcoma incidence and survival in the United States, 1975-2005. Cancer 2009;115:4218-26.

2. Rudzinski ER, Anderson JR, Hawkins DS, et al. The World Health Organization Classification of Skeletal Muscle Tumors in Pediatric Rhabdomyosarcoma: A Report From the Children's Oncology Group. Arch Pathol Lab Med 2015;139:1281-7.

3. Meza JL, Anderson J, Pappo AS, et al. Analysis of prognostic factors in patients with nonmetastatic rhabdomyosarcoma treated on intergroup rhabdomyosarcoma studies III and IV: the Children's Oncology Group. J Clin Oncol 2006;24:3844-51.

4. Oberlin O, Rey A, Lyden E, et al. Prognostic factors in metastatic rhabdomyosarcomas: results of a pooled analysis from United States and European cooperative groups. J Clin Oncol 2008;26:2384-9.

5. Missiaglia E, Williamson D, Chisholm J, et al. PAX3/ FOXO1 fusion gene status is the key prognostic molecular marker in rhabdomyosarcoma and significantly improves current risk stratification. J Clin Oncol 2012;30:1670-7.

6. Little DJ, Ballo MT, Zagars GK, et al. Adult rhabdomyosarcoma: outcome following multimodality treatment. Cancer 2002;95:377-88.

7. Wu HY, Snyder HM 3rd, Womer RB. Genitourinary rhabdomyosarcoma: which treatment, how much, and when? J Pediatr Urol 2009;5:501-6.

8. Crist WM, Anderson JR, Meza JL, et al. Intergroup rhabdomyosarcoma study-IV: results for patients with nonmetastatic disease. J Clin Oncol 2001;19:3091-102.

9. Walterhouse DO, Meza JL, Breneman JC, et al. Local control and outcome in children with localized vaginal rhabdomyosarcoma: a report from the Soft Tissue Sarcoma committee of the Children's Oncology Group.
Pediatr Blood Cancer 2011;57:76-83.

10. Rodeberg DA, Anderson JR, Arndt CA, et al. Comparison of outcomes based on treatment algorithms for rhabdomyosarcoma of the bladder/prostate: combined results from the Children's Oncology Group, German Cooperative Soft Tissue Sarcoma Study, Italian Cooperative Group, and International Society of Pediatric Oncology Malignant Mesenchymal Tumors Committee. Int J Cancer 2011;128:1232-9.

11. Malempati S, Hawkins DS. Rhabdomyosarcoma: review of the Children's Oncology Group (COG) Soft-Tissue Sarcoma Committee experience and rationale for current COG studies. Pediatr Blood Cancer 2012;59:5-10.

12. Ghirardi V, Bizzarri N, Guida F, et al. Role of surgery in gynaecological sarcomas. Oncotarget 2019;10:2561-75.

13. Castagnetti M, Herbst KW, Esposito C. Current treatment of pediatric bladder and prostate rhabdomyosarcoma (bladder preserving vs. radical cystectomy). Curr Opin Urol 2019;29:487-92.

14. Minard-Colin V, Walterhouse D, Bisogno G, et al. Localized vaginal/uterine rhabdomyosarcoma-results of a pooled analysis from four international cooperative groups. Pediatr Blood Cancer 2018;65:e27096.

15. Angelini L, Bisogno G, Esposito C, et al. Appraisal of the role of radical prostatectomy for rhabdomyosarcoma in children: oncological and urological outcome. Ther Adv Urol 2018;10:189-96.

16. Walterhouse DO, Pappo AS, Meza JL, et al. Reduction of cyclophosphamide dose for patients with subset 2 lowrisk rhabdomyosarcoma is associated with an increased risk of recurrence: A report from the Soft Tissue Sarcoma Committee of the Children's Oncology Group. Cancer 2017;123:2368-75.

17. Casey DL, Chi YY, Donaldson SS, et al. Increased local failure for patients with intermediate-risk rhabdomyosarcoma on ARST0531: A report from the Children's Oncology Group. Cancer 2019;125:3242-8.

18. Bisogno G, De Salvo GL, Bergeron C, et al. Vinorelbine and continuous low-dose cyclophosphamide as maintenance chemotherapy in patients with highrisk rhabdomyosarcoma (RMS 2005): a multicentre, open-label, randomised, phase 3 trial. Lancet Oncol 2019;20:1566-75.

19. Weigel BJ, Lyden E, Anderson JR, et al. Intensive Multiagent Therapy, Including Dose-Compressed Cycles of Ifosfamide/Etoposide and Vincristine/ Doxorubicin/Cyclophosphamide, Irinotecan, and Radiation, in Patients With High-Risk 
Rhabdomyosarcoma: A Report From the Children's Oncology Group. J Clin Oncol 2016;34:117-22.

20. Lawrence W Jr, Anderson JR, Gehan EA, et al. Pretreatment TNM staging of childhood rhabdomyosarcoma: a report of the Intergroup Rhabdomyosarcoma Study Group. Children's Cancer Study Group. Pediatric Oncology Group. Cancer 1997;80:1165-70.

21. Maurer HM, Beltangady M, Gehan EA, et al. The Intergroup Rhabdomyosarcoma Study-I. A final report . Cancer 1988;61:209-20.

22. Crist W, Gehan EA, Ragab AH, et al. The Third Intergroup Rhabdomyosarcoma Study. J Clin Oncol 1995;13:610-30.

23. Sultan I, Ferrari A. Selecting multimodal therapy for rhabdomyosarcoma. Expert Rev Anticancer Ther 2010;10:1285-301.

24. Williamson D, Missiaglia E, de Reynies A, et al. Fusion gene-negative alveolar rhabdomyosarcoma is clinically and molecularly indistinguishable from embryonal rhabdomyosarcoma. J Clin Oncol 2010;28:2151-8.

25. Skapek SX, Anderson J, Barr FG, et al. PAX-FOXO1 fusion status drives unfavorable outcome for children with rhabdomyosarcoma: a children's oncology group report. Pediatr Blood Cancer 2013;60:1411-7.

26. Sorensen PHB, Lynch JC, Qualman SJ, et al. PAX3FKHR and PAX7-FKHR gene fusions are prognostic indicators in alveolar rhabdomyosarcoma: A report from the children's oncology group. J Clin Oncol 2002;20:2672-9.

27. Hibbitts E, Chi YY, Hawkins DS, et al. Refinement of risk stratification for childhood rhabdomyosarcoma using FOXO1 fusion status in addition to established clinical outcome predictors: A report from the Children's Oncology Group. Cancer Med 2019;8:6437-48.

28. Mascarenhas L, Chi YY, Hingorani P, et al. Randomized Phase II Trial of Bevacizumab or Temsirolimus in Combination With Chemotherapy for First Relapse Rhabdomyosarcoma: A Report From the Children's Oncology Group. J Clin Oncol 2019;37:2866-74

29. Gallego S, Zanetti I, Orbach D, et al. Fusion status in patients with lymph node-positive (N1) alveolar rhabdomyosarcoma is a powerful predictor of prognosis: Experience of the European Paediatric Soft Tissue Sarcoma Study Group (EpSSG). Cancer 2018;124:3201-9.

30. Casey DL, Wexler LH, Pitter KL, et al. Genomic Determinants of Clinical Outcomes in Rhabdomyosarcoma. Clin Cancer Res 2020;26:1135-40.
31. Hingorani P, Missiaglia E, Shipley J, et al. Clinical Application of Prognostic Gene Expression Signature in Fusion Gene-Negative Rhabdomyosarcoma: A Report from the Children's Oncology Group. Clin Cancer Res 2015;21:4733-9.

32. Borinstein SC, Steppan D, Hayashi M, et al. Consensus and controversies regarding the treatment of rhabdomyosarcoma. Pediatr Blood Cancer 2018. doi: 10.1002/pbc.26809.

33. Dénes FT, Duarte RJ, Cristofani LM, et al. Pediatric genitourinary oncology. Front Pediatr 2013;1:48.

34. Harel M, Ferrer FA, Shapiro LH, et al. Future directions in risk stratification and therapy for advanced pediatric genitourinary rhabdomyosarcoma. Urol Oncol 2016;34:103-15.

35. Bougeard G, Renaux-Petel M, Flaman JM, et al. Revisiting Li-Fraumeni Syndrome From TP53 Mutation Carriers. J Clin Oncol 2015;33:2345-52.

36. Palmero EI, Achatz MI, Ashton-Prolla P, et al. Tumor protein 53 mutations and inherited cancer: beyond LiFraumeni syndrome. Curr Opin Oncol 2010;22:64-9.

37. Kerr B, Delrue MA, Sigaudy S, et al. Genotype-phenotype correlation in Costello syndrome: HRAS mutation analysis in 43 cases. J Med Genet 2006;43:401-5.

38. Stewart DR, Best AF, Williams GM, et al. Neoplasm Risk Among Individuals With a Pathogenic Germline Variant in DICER1. J Clin Oncol 2019;37:668-76.

39. Zangari A, Zaini J, Gulia C. Genetics of Bladder Malignant Tumors in Childhood. Curr Genomics 2016;17:14-32.

40. Kesserwan C, Friedman Ross L, Bradbury AR, et al. The Advantages and Challenges of Testing Children for Heritable Predisposition to Cancer. Am Soc Clin Oncol Educ Book 2016;35:251-69.

41. Cecchetto G, Bisogno G, De Corti F, et al. Biopsy or debulking surgery as initial surgery for locally advanced rhabdomyosarcomas in children?: the experience of the Italian Cooperative Group studies. Cancer 2007;110:2561-7.

42. Shapiro DD, Harel M, Ferrer F, et al. Focusing on organ preservation and function: paradigm shifts in the treatment of pediatric genitourinary rhabdomyosarcoma. Int Urol Nephrol 2016;48:1009-13.

43. Seitz G, Fuchs J, Sparber-Sauer M, et al. Improvements in the Treatment of Patients Suffering from Bladder-Prostate Rhabdomyosarcoma: A Report from the CWS-2002P Trial. Ann Surg Oncol 2016;23:4067-72.

44. Rogers T, Minard-Colin V, Cozic N, et al. Paratesticular rhabdomyosarcoma in children and adolescents-Outcome 
and patterns of relapse when utilizing a nonsurgical strategy for lymph node staging: Report from the International Society of Paediatric Oncology (SIOP) Malignant Mesenchymal Tumour 89 and 95 studies. Pediatr Blood Cancer 2017. doi: 10.1002/pbc.26486.

45. Walterhouse DO, Barkauskas DA, Hall D, et al. Demographic and Treatment Variables Influencing Outcome for Localized Paratesticular Rhabdomyosarcoma: Results From a Pooled Analysis of North American and European Cooperative Groups. J Clin Oncol 2018;36:JCO2018789388.

46. Dang ND, Dang PT, Samuelian J, et al. Lymph node management in patients with paratesticular rhabdomyosarcoma: a population-based analysis. Cancer 2013;119:3228-33.

47. Stevens MC, Rey A, Bouvet N, et al. Treatment of nonmetastatic rhabdomyosarcoma in childhood and adolescence: third study of the International Society of Paediatric Oncology--SIOP Malignant Mesenchymal Tumor 89. J Clin Oncol 2005;23:2618-28.

48. Martelli H, Oberlin O, Rey A, et al. Conservative treatment for girls with nonmetastatic rhabdomyosarcoma of the genital tract: A report from the Study Committee of the International Society of Pediatric Oncology. J Clin Oncol 1999;17:2117-22.

49. Gerbaulet AP, Esche BA, Haie CM, et al. Conservative treatment for lower gynecological tract malignancies in children and adolescents: the Institut Gustave-Roussy experience. Int J Radiat Oncol Biol Phys 1989;17:655-8.

50. Wolden SL, Lyden ER, Arndt CA, et al. Local Control for Intermediate-Risk Rhabdomyosarcoma: Results From D9803 According to Histology, Group, Site, and Size: A Report From the Children's Oncology Group. Int J Radiat Oncol Biol Phys 2015;93:1071-6.

51. Breneman J, Meza J, Donaldson SS, et al. Local control with reduced-dose radiotherapy for lowrisk rhabdomyosarcoma: a report from the Children's Oncology Group D9602 study. Int J Radiat Oncol Biol Phys 2012;83:720-6.

52. Malempati S, Rodeberg DA, Donaldson SS, et al. Rhabdomyosarcoma in infants younger than 1 year: a report from the Children's Oncology Group. Cancer 2011;117:3493-501.

53. Orbach D, Rey A, Oberlin O, et al. Soft tissue sarcoma or malignant mesenchymal tumors in the first year of life: experience of the International Society of Pediatric Oncology (SIOP) Malignant Mesenchymal Tumor Committee. J Clin Oncol 2005;23:4363-71.
54. Bradley JA, Kayton ML, Chi YY, et al. Treatment Approach and Outcomes in Infants With Localized Rhabdomyosarcoma: A Report From the Soft Tissue Sarcoma Committee of the Children's Oncology Group. Int J Radiat Oncol Biol Phys 2019;103:19-27.

55. Ferrari A, Casanova M, Bisogno G, et al. Rhabdomyosarcoma in infants younger than one year old: a report from the Italian Cooperative Group. Cancer 2003;97:2597-604.

56. Million L, Anderson J, Breneman J, et al. Influence of noncompliance with radiation therapy protocol guidelines and operative bed recurrences for children with rhabdomyosarcoma and microscopic residual disease: a report from the Children's Oncology Group. Int J Radiat Oncol Biol Phys 2011;80:333-8.

57. Donaldson SS, Meza J, Breneman JC, et al. Results from the IRS-IV randomized trial of hyperfractionated radiotherapy in children with rhabdomyosarcoma--a report from the IRSG. Int J Radiat Oncol Biol Phys 2001;51:718-28.

58. Martelli H, Haie-Meder C, Branchereau S, et al. Conservative surgery plus brachytherapy treatment for boys with prostate and/or bladder neck rhabdomyosarcoma: a single team experience. J Pediatr Surg 2009;44:190-6.

59. Sardaro A, Carbonara R, Petruzzelli MF, et al. Proton therapy in the most common pediatric non-central nervous system malignancies: an overview of clinical and dosimetric outcomes. Ital J Pediatr 2019;45:170.

60. Breneman JC, Donaldson SS, Constine L, et al. The Children's Oncology Group Radiation Oncology Discipline: 15 Years of Contributions to the Treatment of Childhood Cancer. Int J Radiat Oncol Biol Phys 2018;101:860-74.

61. Leiser D, Calaminus G, Malyapa R, et al. Tumour control and Quality of Life in children with rhabdomyosarcoma treated with pencil beam scanning proton therapy. Radiother Oncol 2016;120:163-8.

62. Cotter SE, Herrup DA, Friedmann A, et al. Proton radiotherapy for pediatric bladder/prostate rhabdomyosarcoma: clinical outcomes and dosimetry compared to intensity-modulated radiation therapy. Int J Radiat Oncol Biol Phys 2011;81:1367-73.

63. Rodeberg DA, Wharam MD, Lyden ER, et al. Delayed primary excision with subsequent modification of radiotherapy dose for intermediaterisk rhabdomyosarcoma: a report from the Children's Oncology Group Soft Tissue Sarcoma Committee. Int J 
Cancer 2015;137:204-11.

64. Heyn RM, Holland R, Newton WA, et al. The role of combined chemotherapy in the treatment of rhabdomyosarcoma in children. Cancer 1974;34:2128-42.

65. Gallego S, Llort A, Roma J, et al. Detection of bone marrow micrometastasis and microcirculating disease in rhabdomyosarcoma by a real-time RT-PCR assay. Journal of Cancer Research and Clinical Oncology 2006;132:356-62.

66. Hawkins DS, Chi YY, Anderson JR, et al. Addition of Vincristine and Irinotecan to Vincristine, Dactinomycin, and Cyclophosphamide Does Not Improve Outcome for Intermediate-Risk Rhabdomyosarcoma: A Report From the Children's Oncology Group. J Clin Oncol 2018;36:2770-7

67. Walterhouse DO, Pappo AS, Meza JL, et al. Shorterduration therapy using vincristine, dactinomycin, and lower-dose cyclophosphamide with or without radiotherapy for patients with newly diagnosed lowrisk rhabdomyosarcoma: a report from the Soft Tissue Sarcoma Committee of the Children's Oncology Group. J Clin Oncol 2014;32:3547-52.

68. Rich TA, Kirichenko AV. Camptothecin dose, schedule, and timing of administration for clinical radiation sensitization. Ann N Y Acad Sci 2000;922:334-9.

69. Arndt CA, Stoner JA, Hawkins DS, et al. Vincristine, actinomycin, and cyclophosphamide compared with vincristine, actinomycin, and cyclophosphamide alternating with vincristine, topotecan, and cyclophosphamide for intermediate-risk rhabdomyosarcoma: children's oncology group study D9803. J Clin Oncol 2009;27:5182-8.

70. Bisogno G, Jenney M, Bergeron C, et al. Addition of dose- intensified doxorubicin to standard chemotherapy for rhabdomyosarcoma (EpSSG RMS 2005): a multicentre, open-label, randomised controlled, phase 3 trial. Lancet Oncol 2018;19:1061-71.

71. Perkins SM, Shinohara ET, DeWees T, et al. Outcome for children with metastatic solid tumors over the last four decades. PLoS One 2014;9:e100396.

72. Gupta AA, Chi YY, Anderson JR, et al. Patterns of chemotherapy-induced toxicities and outcome in children and adolescents with metastatic rhabdomyosarcoma: A report from the Children's Oncology Group. Pediatr Blood Cancer 2017;64:10.1002/pbc.26479.

73. Ballinger ML, Goode DL, Ray-Coquard I, et al. Monogenic and polygenic determinants of sarcoma risk: an international genetic study. Lancet Oncol 2016;17:1261-71.

74. Kim A, Widemann BC, Krailo M, et al. Phase 2 trial of sorafenib in children and young adults with refractory solid tumors: A report from the Children's Oncology Group. Pediatr Blood Cancer 2015;62:1562-6.

75. Pappo AS, Vassal G, Crowley JJ, et al. A phase 2 trial of R1507, a monoclonal antibody to the insulin-like growth factor-1 receptor (IGF-1R), in patients with recurrent or refractory rhabdomyosarcoma, osteosarcoma, synovial sarcoma, and other soft tissue sarcomas: results of a Sarcoma Alliance for Research Through Collaboration study. Cancer 2014;120:2448-56.

76. Wagner LM, Fouladi M, Ahmed A, et al. Phase II study of cixutumumab in combination with temsirolimus in pediatric patients and young adults with recurrent or refractory sarcoma: a report from the Children's Oncology Group. Pediatr Blood Cancer 2015;62:440-4.
Cite this article as: Deel MD. Advances in the management of pediatric genitourinary rhabdomyosarcoma. Transl Androl Urol 2020;9(5):2441-2454. doi:10.21037/tau-20-480 